\title{
Decoding Xing-Ling codes
}

\author{
Nielsen, Rasmus Refslund
}

Published in:

2002 IEEE International Symposium on Information Theory, 2002. Proceedings.

Link to article, DOI:

10.1109/ISIT.2002.1023360

Publication date:

2002

Document Version

Publisher's PDF, also known as Version of record

Link back to DTU Orbit

Citation (APA):

Nielsen, R. R. (2002). Decoding Xing-Ling codes. In 2002 IEEE International Symposium on Information Theory, 2002. Proceedings. IEEE. https://doi.org/10.1109/ISIT.2002.1023360

\section{General rights}

Copyright and moral rights for the publications made accessible in the public portal are retained by the authors and/or other copyright owners and it is a condition of accessing publications that users recognise and abide by the legal requirements associated with these rights.

- Users may download and print one copy of any publication from the public portal for the purpose of private study or research.

- You may not further distribute the material or use it for any profit-making activity or commercial gain

- You may freely distribute the URL identifying the publication in the public portal

If you believe that this document breaches copyright please contact us providing details, and we will remove access to the work immediately and investigate your claim. 


\title{
Decoding Xing-Ling codes
}

\author{
R. Refslund Nielsen ${ }^{1}$ \\ Research Center COM, building 371 \\ Technical University of Denmark (DTU) \\ DK-2800 Lyngby, Denmark. \\ E-mail: rrnøcom.dtu.dk.
}

\begin{abstract}
This paper describes an efficient decoding method for a recent construction of good linear codes as well as an extension to the construction. Furthermore, asymptotic properties and list decoding of the codes are discussed.
\end{abstract}

\section{XING-Ling CODES}

In [1] Xing and Ling describes a new construction of a class of linear codes (here referred to as Xing-Ling codes) which resulted in several improvements to Brouwer's table [2] of good linear codes.

A Xing-Ling code is a subfield subcode of a Reed-Solomon code over $\mathbb{F}_{q^{2}}$. While a Reed-Solomon code of dimension $K$ is obtained by evaluating elements of $\mathbb{F}_{q^{2}}$ in all polynomials of degree at most $K-1$, a Xing-Ling code is obtained by evaluating certain elements of $\mathbb{F}_{q^{2}}$ in certain polynomials of degree at most $K-1$. The elements and polynomials are chosen in such a way that the result is a code over $\mathbb{F}_{q}$.

For any integer, $K$, let $V_{K}$ denote the $\mathbb{F}_{q}$-vector space spanned by all monomials and monic binomials of degree less than $K$ which only give values in $\mathbb{F}_{q}$ when evaluated in elements from $\mathbb{F}_{q^{2}}$.

We then have the following definition of Xing-Ling codes:

Definition 1 Let $A \subseteq \mathbb{F}_{q}$ and $B \subseteq \mathbb{F}_{q^{2}} \backslash \mathbb{F}_{q}$ be given such that $\beta^{q} \notin B$ for all $\beta \in B$ and let $K$ be given such that $V_{K} \neq V_{K-1}$. Then the following set is a Xing-Ling code:

$$
X L(A, B, K):=\left\{f(A, B) \mid f \in V_{K}\right\}
$$

The main parameters of Xing-Ling codes are summarized in the following theorem:

Theorem 2 ([1] , Theorem 2.5, 2.6, 2.9, and 2.10) The code $X L(A, B, K)$ satisfies the following:

1. The code is a linear code over $\mathbb{F}_{q}$.

2. Let the number of elements of $A$ and $B$ be denoted by

$$
n_{A}:=|A| \quad \text { and } \quad n_{B}:=|B| .
$$

The length of the code is then $n=n_{A}+n_{B}$ and if $K-1=q r+s$ where $0 \leq s \leq q$ then the dimension is $k=(r(r+1)) / 2+s+1$.

3. Let

$$
z:= \begin{cases}\max \{2(r-1), r+s\} & \text { if } q \text { is odd } \\ \max \{r-1, s\} & \text { if } q \text { is even. }\end{cases}
$$

Then the minimum distance, $d$, satisfies $d \geq d^{*}$ where

$$
d^{*}:=n-\left\lfloor\frac{K-1+\max \left\{\min \left\{z, n_{A}\right\}, 2 n_{A}-\delta q\right\}}{2}\right\rfloor
$$

with $\delta=2$ for $q$ odd and $\delta=1$ for $q$ even. Notice that for $q$ odd the first term of the max-expression is always largest since $n_{A} \leq q$.

${ }^{1}$ This work was done at Department of Mathematics, DTU.
In the definition of Xing-Ling codes we have the constraints $n_{A} \leq q$ and $n_{B} \leq\left(q^{2}-q\right) / 2$ so the length of a Xing-Ling code is at most $q(q+1) / 2$. However, the paper [3] describes how to extend the code with one position by evaluating in the point at infinity on the projective line. This gives a few improvements to Brouwer's table.

\section{DECODING}

Suppose that a word $r \in \mathbb{F}_{q}^{n}$ is received. The goal is to find the polynomial $\hat{f} \in V_{K}$ such that $\hat{f}$ corresponds to the XingLing codeword closest to $r$. The paper describes an efficient method that calculates $\hat{f}$ if the corresponding codeword has distance less than half the designed minimum distance from the received word. The method is sketched below.

The word $r$ is decomposed into two blocks, $r=\left(r_{A}, r_{B}\right)$ where $r_{A}$ are the received values on the $A$-positions - the positions corresponding to the set $A-$ and similarly $r_{B}$ are the received values on the $B$-positions.

If $n_{A} \leq z$ (with $z$ defined in Eqn. (1)) then it turns out that it suffices to decode the word $\left(r_{A}, r_{B}, r_{B}\right)$ with a suitable Reed-Solomon code over $\mathbb{F}_{q^{2}}$.

If $n_{A}>z$ then this approach fails if too many errors occurred on the $B$-positions. In that case the word $r_{A}$ is decoded with a Reed-Solomon code over $\mathbb{F}_{q}$. This results in an estimate, $u$, of $\hat{f}\left(\mathbb{F}_{q}\right)$. If $q$ is even then decoding $\left(u, r_{B}, r_{B}\right)$ with a Reed-Solomon code gives the result. If $q$ is odd then the decoding is done with a so-called generalized Reed-Solomon $m$-code which is defined in the paper.

\section{Asymptotic Results}

Let an infinite sequence of Xing-Ling codes be constructed for alphabet sizes tending to infinity such that for each alphabet size, $q$, we have $n_{A}=0$ and $n_{B}=n=\left(q^{2}-q\right) / 2$ and such that the information rate $(k / n)$ tends to a constant, $\kappa$.

For $q \rightarrow \infty$ it is then shown that the designed minimum distance, $d^{*}$, satisfies

$$
d^{*} / n \rightarrow 1-\sqrt{\kappa}
$$

and that a fraction of errors, $t / n \rightarrow \tau$, can be efficiently list decoded whenever

$$
\tau<1-\sqrt{\sqrt{\kappa}}
$$

\section{REFERENCES}

[1] C. Xing and S. Ling: "A Class of Linear Codes with Good Parameters", IEEE Transactions on Information Theory, vol. 46 , no. 6 , pp. $2184-2188,2000$.

[2] A. Brouwer: Bounds on the minimum distance of linear codes. Online. URL: http://www.win.tue.nl/ aeb/voorlincod.html.

[3] R. Refslund Nielsen: "Decoding Xing-Ling codes", submitted for publication in IEEE Transactions on Information Theory, 2001. 